\title{
Influence of cigarette smoking on the outcome of coronary care unit admissions with chest pain
}

\author{
S. Mallya, P.M.A. Calverley, I.A. MacFarlane, S. Hughes, G. Johnston and \\ C. van Heyningen
}

Department of Medicine and Pathology, Aintree Hospitals, Liverpool, UK

\begin{abstract}
Summary: We studied the effect of current smoking habits in the period immediately before admission to hospital in 90 consecutive patients presenting with chest pain, 50 of whom were shown to have myocardial infarction. Urine cotinine/creatinine (cot/creat) ratio measured within 4 hours of admission was used as an objective marker of cigarette smoking in the preceding 18 hours. Fifty-seven patients had urine cot/creat ratios suggesting recent smoking, although four of these denied smoking. Patients with myocardial infarction had higher median cot/creat ratios $(3.31 \mu \mathrm{g} / \mathrm{mg}$, range $0-17.8)$ compared with patients with non-infarct chest pain $(0.5 \mu \mathrm{g} / \mathrm{mg}$, range $0-37.2)$. Sixteen patients with cardiac rhythm disturbances following infarction had significantly higher cot/creat ratios than the 34 infarct patients without this complication (median and range $8.34 \mu \mathrm{g} / \mathrm{mg} ; 0-17.8 \mathrm{~V} .1 .87 \mu \mathrm{g} / \mathrm{mg} ; 0-16.4, P<0.01$ ). Tobacco use in the $\mathbf{2 4}$ hours before myocardial infarction may predispose to cardiac rhythm disturbance, irrespective of infarct size.
\end{abstract}

\section{Introduction}

Cigarette smoking is an important risk factor for ischaemic heart disease and the mortality from myocardial infarction rises as the amount smoked increases. ${ }^{1}$ Conversely smoking cessation reduces the subsequent mortality rate and the incidence of sudden death, irrespective of infarct size. ${ }^{2}$ The gas phase of cigarette smoke is rich in carbon monoxide (CO) with a circulation half-life of approximately 2 hours $^{3}$ which might compromise oxygen delivery to the ischaemic myocardium. The particulate phase of smoke contains $1.3 \mathrm{mg}$ nicotine for a $500 \mathrm{mg}$ delivery cigarette, with welldocumented cardiovascular as well as central nervous system effects. ${ }^{3}$ Recent exposure to either or both of these agents during the early stages of critical myocardial ischaemia might therefore increase the extent of myocardial damage and/or the incidence of cardiac rhythm disturbances. However, objective data about cigarette exposure during this critical period is lacking.

Situations where the psychological pressures to stop smoking are greatest increase the likelihood of an incorrect smoking history. ${ }^{4}$ Admission to hospital with chest pain is a very stressful experience and information about the reliability of the smoking history in this situation is not available.

Correspondence: S. Mallya, M.D., M.R.C.Path., Pathology Department, Walton Hospital, Rice Lane, Liverpool L9 1AE, UK.

Accepted: 5 February 1992
We have investigated these problems by conduc 8 ting a prospective study in a group of patients admitted to our coronary care unit with a history o cardiac chest pain and related their stated smoking habits to the urinary cotinine/creatinine (cot/creat) ratio, a measure of cigarette exposure during the preceding 18 hours. ${ }^{5}$ In addition, we recorded the incidence of significant rhythm disturbances in the first 36 hours after admission and the presence of complications during the hospital stay to see if pre-admission cigarette exposure related to these measures of outcome.

\section{Methods and materials}

We studied 90 consecutive admissions to our hospital coronary care unit (CCU) with a diagnosis of chest pain, thought to be due to cardiac ischaemia. All patients had developed chest pain within 12 hours before admission and patients sustaining a cardiac arrest prior to CCU admission were excluded in this study. Patients were divided into three groups. There were 50 patients with serial electrocardiogram (ECG) and cardiac enzyme studies confirming an acute myocardial infarction, 34 males, mean age 56.8 years (range $37-81$ years), 20 of whom smoked by urine cot/creat ratio. The 16 females had a mean age of 60.5 years (range $46-70$ years) and 13 were smokers. There were 29 with cardiac ischaemia without infarction. Nineteen were males, mean age 52.5 years (range 34-67 
years) and 12 were smokers. Ten were females, mean age 56.6 years (range 33-75 years) and six were smokers. Finally, there were 11 patients with a final diagnosis of non-cardiac chest pain, seven males, mean age 45.4 years (range 31-63 years), five smokers. Four were female, mean age 69.3 years (range 56-83 years), one smoker.

We admitted all patients suspected of acute myocardial infarction to our CCU and used standard ECG criteria of infarction on a 12-lead ECG (Hewlett Packard Pagewriter) to diagnose infarction, whilst ischaemia without infarction was diagnosed in the presence of a history of typical cardiac non-exertional chest pain with or without a positive stress test. The remaining group lacked these features or had clinical or radiographic evidence of musculo-skeletal or gastro-intestinal causes. Among the myocardial infarction group (group 1), 24 were anterior and 26 inferior and six died during this admission. There were no fatalities in the non-infarction group.

A history of current smoking habit was obtained by the admitting physician and an aliquot of urine $(25 \mathrm{ml})$ was obtained when the patient first passed urine (within 4 hours) and was stored at $-20^{\circ} \mathrm{C}$ for subsequent analysis of urinary cotinine and creatinine. A colorimetric diethylthio-barbituric acid (DETB) extraction method was used for cotinine measurement. This method has a lower false positive rate than non-extraction methods. ${ }^{6}$ Patients were monitored for the presence of major complications, i.e. cardiac arrest, the development of clinically significant congestive cardiac failure or cardiogenic shock and significant cardiac rhythm disturbances.

Patients who were diagnosed as having noncardiac chest pain were discharged to the ward within 24 hours whilst myocardial infarction and ischaemic chest pain patients were retained for at least 36 hours of $\mathrm{CCU}$ monitoring. No patient received thrombolytic therapy. This treatment was not routinely used in our coronary care unit at the time of the study.

For comparison we have used data on the smoking habits and cot/creat ratios in 18 smoking and 24 non-smoking healthy laboratory and medical personnel. This data has been reported previously. ${ }^{7}$ Group data are expressed as median with range and comparisons between groups have been made using non-parametric Mann-Whitney $U$-test and Spearman test. This study was approved by the hospital ethical committee.

\section{Results}

Fifty-three of the 90 chest pain patients admitted to being life-long smokers, 28 to be ex-smokers of at least one month's duration, and 9 said they had never smoked. In our control non-smoking group of medical and laboratory personnel, no subject had a urine cot/creat ratio $>1.2 \mu \mathrm{g} / \mathrm{mg}$. Four patients who said they were ex-smokers had a urine cot/creat ratio ranging from 2.57 to $5.64 \mu \mathrm{g} / \mathrm{mg}$ suggesting active smoking in the previous 18 hours. These four patients were therefore included as current smokers in further analysis of data. The cot/creat ratios of the 57 patients who smoked showed a greater variance than was found in the control smoking group (Table I). There was no significant difference in the cot/creat ratio between the two sexes (males median $2.1 \mu \mathrm{g} / \mathrm{mg}$, range $0-37.2$ and females median $4.8 \mu \mathrm{g} / \mathrm{mg}$, range $0-17.8$ ). There was a negative correlation between cot/creat ratio and age $(r=-0.318, P=0.002)$. We found no significant differences between the cot/creat ratios from the 50 patients with documented myocardial infarction (median $3.31 \mu \mathrm{g} /$ $\mathrm{mg}$, range $0-17.8$ ) and those with non-infarction chest pain (median $0.50 \mu \mathrm{g} / \mathrm{mg}$, range $0-37.2$ ) (Figure 1). Among the myocardial infarction patients there was no difference in cot/creat ratios between patients with inferior and anterior myocardial infarction (median cot/creat ratio in

Table I Median and range of urine cotinine/creatinine ratio in healthy control subjects and the patients with chest pain

\begin{tabular}{lcccc}
\hline & \multicolumn{2}{c}{ Control group } & \multicolumn{2}{c}{ Chest pain } \\
& $s m$ & $N$-sm & $s m$ & $N$-sm \\
\hline $\begin{array}{l}\text { Number of } \\
\text { patients }\end{array}$ & 18 & 24 & $57^{*}$ & 33 \\
$\begin{array}{l}\text { Urine cotinine/ } \\
\text { creatinine } \\
\text { ratio }(\mu \mathrm{g} / \mathrm{mg})\end{array}$ & 6.8 & 0.7 & 8.7 & 0.59 \\
\hline
\end{tabular}

$\mathrm{sm}=$ smoker; $\mathrm{N}$-sm $=$ non-smoker; ${ }^{*}$ The 57 patients with chest pain who smoked included four who denied smoking but had a urine cot/creat ratio $>1.2 \mu \mathrm{g} / \mathrm{mg}$. 


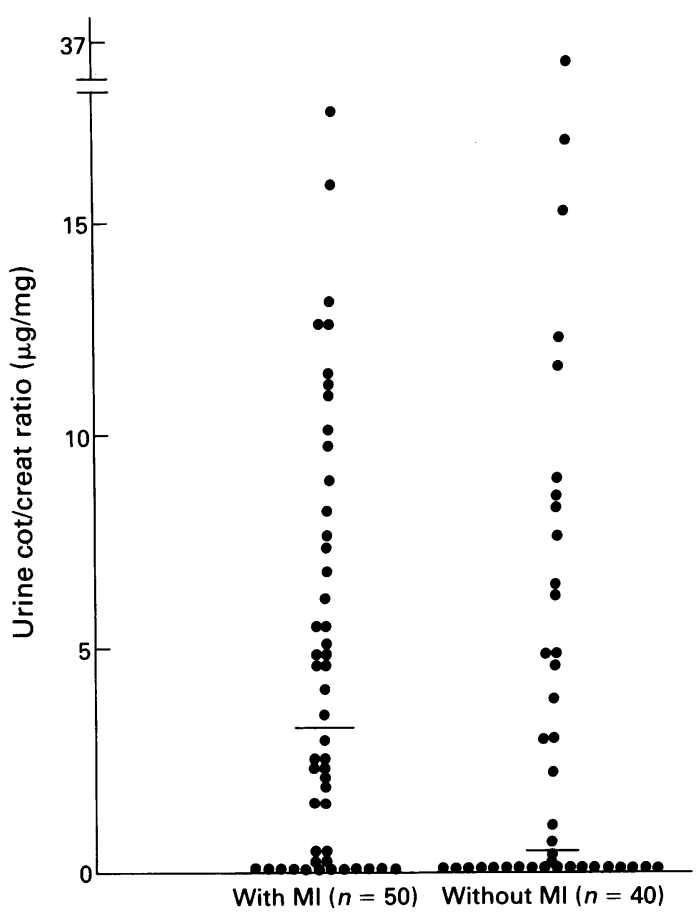

Figure 1 Urine cotinine/creatinine ratio (with median) in patients with and without myocardial infarction (MI).

inferior myocardial infarction $(n=26)=4.03 \mu \mathrm{g}$ / $\mathrm{mg}$ (range $0-17.8$ ): anterior myocardial infarction $(n=24)=4.20 \mu \mathrm{g} / \mathrm{mg}$ (range $0-13.4))$. Of the six patients who died following admission, five were current smokers. The mean urinary cot/creat ratio in this group did not differ significantly from those who survived a myocardial infarction (median cot/creat ratio 3.7 and $3.31 \mu \mathrm{g} / \mathrm{mg}$, respectively).

During the first 36 hours after admission there were five patients with ventricular tachycardia and/or fibrillation, and 11 others with either episodes of atrial fibrillation or prolonged periods of frequent unifocal ventricular ectopics. The six deaths occurred in this group of 16 patients with rhythm disturbance. Only one of these 16 patients was a non-smoker. Six patients had both cardiac rhythm disturbances and clinical or radiological evidence of cardiac failure. The cot/creat ratio of the 16 patients with rhythm disturbances (mean age $53.3 \pm 10.24$ years, eight males, eight females) was significantly greater (median $8.34 \mu \mathrm{g} / \mathrm{mg}$, range $0-17.8$ ) than those patients with myocardial infarction without these rhythm disturbances; $n=34$, mean age $60.1 \pm 10.23$ years, 26 males (13 smokers, 13 non-smokers) and eight females (five smokers and three non-smokers) (median $1.87 \mu \mathrm{g} /$ $\mathrm{mg}$, range $0-16.14)(P<0.01)$ (Figure 2). There was no significant difference in the ages of those with and without rhythm disturbances (unpaired

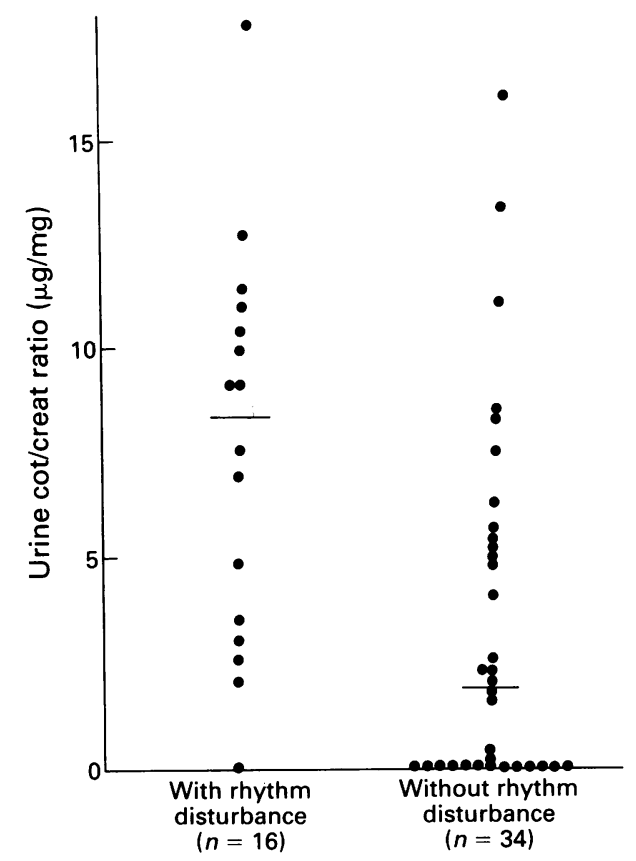

Figure 2 Urine cotinine/creatinine ratio (with median) in patients with myocardial infarction $(P=0.002)$.

$t$-test). The levels of creatine kinase (CK) and aspartate transaminase (AST) at 24 hours did not differ significantly between these groups (myocar- $\frac{}{\infty}$ dial infarction with arrhythmias, median $\mathbf{C K}=\varrho$ $551 \mathrm{U} / 1$, range $54-3,430 ;$ median $\mathrm{AST}=255 \mathrm{U} / 1, \overrightarrow{\overrightarrow{0}}$ range 52-522; myocardial infarction without arr hythmias, median CK $=920 \mathrm{U} / 1$, range $54-3,430$, median AST $=144 \mathrm{U} / 1$, range $25-520$ ).

\section{Discussion}

Although the aetiology of ischaemic heart disease is multifactorial there is convincing evidence that ${ }^{?}$ cigarette smoking changes vascular epithelium, $\frac{8 D}{O}$ promotes atherosclerosis and increases the in cidence of mortality from myocardial infarct ate least in high prevalence areas. ${ }^{9}$ There is cleañ evidence that smoking cessation lessens the sub-N sequent risk of sudden death/myocardial infarction ${ }^{\omega}$ even when infarct type and size are matched. ${ }^{10-12}$ This effect is similar in magnitude to but indepen dent of the secondary protective effects of propranolol. ${ }^{13}$ Smoking enhances the risk of infarction even when subjects are matched for angiographically evident coronary disease but which of the complex components inhaled is most toxic is not yet known. 
Two of the biologically important chemicals inhaled are carbon monoxide and nicotine and both have been used as markers of cigarette consumption. Carbon monoxide inhalation is associated with a reduced effort tolerance in angina, even at relatively modest exposure. ${ }^{14}$ The total carbon monoxide exposure varies with the type of cigarette, whilst individuals smoke to maintain plasma nicotine at a constant level. ${ }^{15,16}$ Nicotine is metabolized in the liver and excreted as the stable metabolite cotinine. Urine cotinine is a good marker of tobacco exposure over the preceding 18 hours when normalized for urine creatinine ${ }^{13,17}$ Breath carbon monoxide concentration however reflect exposure only for the last 2 hours.

This report highlights several important problems. Despite the stressful nature of the illness, we found four patients who denied smoking but had abnormal urine tests. It is possible that some of the wide variation we saw in cot/creat ratio is due to under-reporting of true cigarette use.

The cotinine levels did not distinguish infarction from patients with ischaemia alone. However this is a small series which lacks the statistical power to exclude such an effect. More impressive were the higher than average cot/creat ratios among the patients dying of infarction whose CK/AST did not differ from the non-fatal group. Once more interpretation of these findings must be cautious as the interpretation of two cardiac enzymes measured within the first 24 hours of admission may underestimate true differences in infarct size but we believe these findings are worth further study.

Among the infarct patients with tachy-rhythm disturbances, the cot/creat ratios were significantly greater than those without such arrhythmias. Details of cotinine levels were not available to the staff monitoring the patients. These changes may reflect the effects of nicotine on the release of catecholamines, heart rate and blood pressure. ${ }^{18}$ It is likely to be an independent pro-arrhythmic factor as catecholamine release is increased significantly during infarction and local ischaemia would predispose to these nicotine-related effects.

In summary these data suggest that the measurement of urinary cot/creat ratio can provide valuable additional information about recent smoking habits in patients admitted with cardiac chest pain. The dangers associated with tobacco smoke may not simply be those of promoting coronary atheroma but it may lead to potentially life-threatening rhythm disturbances. The prevalence of these smoking-related problems during acute ischaemia merits further investigation.

\section{Acknowledgement}

The authors wish to thank Mrs C. Ganley for her help with the typing of this manuscript.

\section{References}

1. Department of Health, Education and Welfare. Smoking and Health: A report of the Surgeon General. Government Printing Office, Washington, DC, 1979.

2. Cohen, A.J. \& Roe, F.J.C. Monograph on the pharmacology and toxicology of nicotine. Tobacco Advisory Council Occasional Paper 4, London, 1981, p. 45.

3. Huber, G.L. Physical, chemical and biologic properties of tobacco, cigarette smoke and other tobacco products. Sem Resp Med 1989, 10: 297-327.

4. Heyston-Miller, N., Haskell, W.L. \& De Busk, R.F. Smoking cessation after acute myocardial infarction: the effects of exercise training. Addict Behav 1989, 13: 331-335.

5. Hill, P. \& Marquardt, H. Plasma and urine changes after smoking different brands of cigarettes. Clin Pharmacol Therap 1980, 27: 652-658.

6. Peach, H., Ellard, G.A., Jenner, P.J. \& Morris, R.W. A simple inexpensive test of smoking. Thorax 40: 351-357.

7. Ardon, M., MacFarlane, I.A., Robinson, C., Van Heyningen, C. \& Calverley, P.M.A. Anti-smoking advice for young diabetic smokers: Is it a waste of breath? Diabet Med 1988, 5: 667-670.

8. Booyse, F.M., Osikowicz, G. \& Quarfoot, A.J. Effects of chronic oral consumption of nicotine on the rabbit aortic endothelium. Am J Path 1981, 102: 229-238.

9. Strong, J.P., Solberg, L.A. \& Restrepo, C. Atherosclerosis in persons with coronary heart disease. Lab Invest 1968, 18: 527-537.

10. Hermanson, B., Omenn, G.S., Kronmal, R.A. \& Gersh, B.J. Beneficial six year outcome of smoking cessation in older men and women with coronary artery disease. $N$ Engl J Med 1988 , 319: $1365-1369$.
11. Robinson, K., Conroy, R.M. \& Mucahy, R. When does the risk of acute coronary heart disease in ex-smokers fall to that of smokers? A retrospective study of patients admitted to hospital with a first episode of myocardial infarction or unstable angina. Br Heart J 1989, 62: 16-29.

12. Rosenberg, L., Palmer, J.R. \& Shapiro, S. Decline in the risk of myocardial infarction among women who stop smoking. $N$ Engl J Med 1990, 322: 213-217.

13. Jafri, S.M., Tilley, B.C., Peters, R., Schultz, L.R. \& Goldstein, $\mathrm{S}$. The effects of cigarette smoking and propranolol in survivors of acute myocardial infarction. Am J Cardiol 1990, 65: 271-276.

14. Anderson, E.W., Andelman, R.J., Strauch, C.F., Fortuin, N.J. \& Knelson, J.H. Effects of low level carbon monoxide exposure on onset and duration of angina pectoris. Ann Intern Med 1973, 79: 46-50.

15. Wald, N., Idle, M., Smith, P.G. \& Bailey, A. Carboxyhaemoglobin levels in smokers of filter and plain cigarettes. Lancet 1977, 2: 110-112.

16. Kaufman, D.W., Helmrich, S.P., Rosenberg, L., Miettinen, O.S. \& Shapiro, S. Nicotine and carbon monoxide content of cigarette smoke and the risk of myocardial infarction in young men. $N$ Engl J Med 1983, 308: 409-413.

17. Boyland, E. \& De Kock, D.H. Nicotine metabolism. British Empire Cancer Campaign for Research, 44th Annual Report. Part II, 5-6, 1981.

18. Cryer, P.E., Haymond, M.W., Santiago, J.V. \& Shah, S.D. Norepinephrine and epinephrine release and adrenergic mediation of smoking-associated haemodynamic and metabolic events. $N$ Engl J Med 1976, 295: 573-577. 